\title{
On the diameter of lattice polytopes
}

\author{
Alberto Del Pia* \\ Carla Michini ${ }^{\dagger}$
}

September 17, 2018

\begin{abstract}
In this paper we show that the diameter of a $d$-dimensional lattice polytope in $[0, k]^{n}$ is at most $\left\lfloor\left(k-\frac{1}{2}\right) d\right\rfloor$. This result implies that the diameter of a $d$-dimensional half-integral polytope is at most $\left\lfloor\frac{3}{2} d\right\rfloor$. We also show that for half-integral polytopes the latter bound is tight for any $d$.
\end{abstract}

\section{Introduction}

The 1-skeleton of a polyhedron $P$ is the graph whose nodes are the vertices of $P$, and that has an edge joining two nodes if and only if the corresponding vertices of $P$ are adjacent on $P$. Given vertices $u, v$ of $P$, the distance $\delta^{P}(u, v)$ between $u$ and $v$ is the length of a shortest path connecting $u$ and $v$ on the 1-skeleton of $P$. We may write $\delta(u, v)$ instead of $\delta^{P}(u, v)$ when the polyhedron we are referring to is clear from the context. The diameter $\delta(P)$ of $P$ is the smallest number that bounds the distance between any pair of vertices of $P$.

In this paper, we investigate the diameter of lattice polytopes, i.e. polytopes whose vertices are integral. Lattice polytopes play a crucial role in discrete optimization and integer programming problems, where the variables are constrained to assume integer values. Our goal is to define a bound on the diameter of a lattice polytope $P$, that depends on the dimension of $P$ and on the parameter $k=\max \left\{\|x-y\|_{\infty}\right.$ : $x, y \in P\}$, in order to apply such bound to classes of polytopes for which $k$ is known to be small. A similar approach has been followed by Bonifas et al. 4, who showed that the diameter of a polyhedron $P=\left\{x \in \mathbb{R}^{n}: A x \leq b\right\}$ is bounded by a polynomial that depends on $n$ and on the parameter $\Delta$, defined as the largest absolute value of any sub-determinant of $A$. Note that, while $\Delta$ is related to the external description of $P, k$ is related to its internal description. However, both $\Delta$ and $k$ are in general not polynomial in $n$ and in the number of the facet-defining inequalities of $P$.

For $k \in \mathbb{N}$, a $(0, k)$-polytope $P \subseteq \mathbb{R}^{n}$ is a lattice polytope contained in $[0, k]^{n}$. Naddef [10] showed that the diameter of a $d$-dimensional $(0,1)$-polytope is at most $d$, and this bound is tight for the hypercube $[0,1]^{d}$. Kleinschmidt and Onn [8] extended this result by proving that the diameter of a $d$-dimensional $(0, k)$-polytope cannot exceed $k d$. However, their bound is not tight for $k \geq 2$.

Our main contribution is establishing an upper bound for the diameter of a $d$-dimensional $(0, k)$ polytope, which refines the bound by Kleinschmidt and Onn.

Theorem 1. For $k \geq 2$, the diameter of a d-dimensional $(0, k)$-polytope is at most $\left\lfloor\left(k-\frac{1}{2}\right) d\right\rfloor$.

The proof of Theorem 1 is elementary, as it combines an induction argument with basic tools from linear programming and polyhedral theory. Our proof is also constructive, since it shows how to build a path between two given vertices of $P$, whose length does not exceed our bound.

For (0,2)-polytopes, we show that the upper bound given in Theorem 1 is tight for any $d$.

Corollary 2. The diameter of a d-dimensional $(0,2)$-polytope is at most $\left\lfloor\frac{3}{2} d\right\rfloor$. Moreover, for any natural number $d$, there exists a d-dimensional $(0,2)$-polytope attaining this bound.

The lower bound of Corollary 2 follows by an easy construction based on the cartesian product of polytopes of dimension one and two. It is well-known that, given two polytopes $P_{1}$ and $P_{2}$, their cartesian product $P_{1} \times P_{2}$ satisfies $\delta\left(P_{1} \times P_{2}\right)=\delta\left(P_{1}\right)+\delta\left(P_{2}\right)$. Now, let $H_{1}=[0,2]$ and $H_{2}=$ $\operatorname{conv}\{(0,0),(1,0),(0,1),(2,1),(1,2),(2,2)\}$. For even $d$, let $H_{d}=\left(H_{2}\right)^{d / 2}$, and for odd $d$, let $H_{d}=$ $H_{d-1} \times H_{1}$. Thus for all $d \in \mathbb{N}, H_{d}$ is a $d$-dimensional $(0,2)$-polytope, with $\delta\left(H_{d}\right)=\left\lfloor\frac{3}{2} d\right\rfloor$.

\footnotetext{
${ }^{*}$ Department of Industrial and Systems Engineering, University of Wisconsin-Madison. Email: delpia@wisc.edu

†Wisconsin Institute for Discovery, University of Wisconsin-Madison. Email: michini@wisc.edu
} 
Corollary 2 has important implications for the diameter of half-integral polytopes. Half-integral polytopes are polytopes whose vertices have components in $\left\{0, \frac{1}{2}, 1\right\}$, and they are affinely equivalent to $(0,2)$-polytopes. The class of half-integral polytopes is very rich, as many half-integral polytopes appear in the literature as relaxations of $(0,1)$-polytopes arising from combinatorial optimization problems. In some cases, while the $(0,1)$-polytope defined as the convex hull of the feasible solutions to the combinatorial problem has exponentially many facets, there is a linear relaxation, defined by a polynomial number of constraints, that yields a half-integral polytope.

There are several classes of polytopes that are known to be half-integral, such as the fractional matching polytope and the fractional stable set polytope [2, the linear relaxation of the boolean quadric polytope and the rooted semimetric polytope [12] (see also [14 and 9]). An interesting class of halfintegral polytopes arises from totally dual half-integral systems, such as the fractional stable matching polytope [1, 6], and the fractional matroid matching polytope [13, 7].

The rest of the paper is devoted to proving Theorem 1 .

\section{Proof of main result}

In order to bound the diameter of a non full-dimensional $(0, k)$-polytope $P \subseteq \mathbb{R}^{n}$, we define the projection of $P$ onto the $i$-coordinate hyperplane as the polytope

$$
\left\{\bar{x} \in \mathbb{R}^{n-1}: \exists x \in P \text { with } x_{j}=\bar{x}_{j} \text { for } j=1, \ldots, i-1, x_{j}=\bar{x}_{j-1} \text { for } j=i+1, \ldots, n\right\} .
$$

That is, we simply drop the $i$-th coordinate from all vectors in $P$. Since integral vectors are mapped into integral vectors, the next lemma follows from Theorem 3.3 in [11].

Lemma 1. Let $P$ be a d-dimensional $(0, k)$-polytope in $\mathbb{R}^{n}$ with $d \geq 1$. Then there exists a fulldimensional $(0, k)$-polytope in $\mathbb{R}^{d}$ with the same 1 -skeleton as $P$.

For $d, k \in \mathbb{N}$, we define $\delta_{k}^{d}$ to be the maximum possible diameter of a $(0, k)$-polytope of dimension at most $d$, i.e.

$$
\delta_{k}^{d}=\max \{\delta(P): P \text { is a }(0, k) \text {-polytope of dimension at most } d\} .
$$

Note that the maximum in the definition of $\delta_{k}^{d}$ always exists. In fact, it follows from Lemma 1 that the number of vertices of a $d$-dimensional $(0, k)$-polytope is at most $(k+1)^{d}$, thus also its diameter is upper bounded by $(k+1)^{d}$, which is a number independent on the dimension of the ambient space of $P$. Moreover, for fixed $k$, the value $\delta_{k}^{d}$ is clearly non-decreasing in $d$.

We now present some lemmas that will be used to prove Theorem 1 . These results follow by applying the ideas introduced by Kleinschmidt and Onn in [8. The next lemma shows how to bound the distance $\delta(u, F)$ between a vertex $u$ of a lattice polytope $P$ and a face $F$ of $P$, that is defined as $\delta(u, F)=$ $\min \{\delta(u, v): v$ is a vertex of $F\}$. We say that two vertices $u, v$ of a polytope are neighbors if $\delta(u, v)=1$. We denote by $e^{i}$, for $i=1, \ldots, n$, the $i$-th vector of the standard basis of $\mathbb{R}^{n}$.

Lemma 2. Let $P$ be a lattice polytope, and let $u$ be a vertex of $P$. Let $c$ be an integral vector, $\gamma=$ $\min \{c x: x \in P\}$, and $F=\{x \in P: c x=\gamma\}$. Then $\delta(u, F) \leq c u-\gamma$.

Proof. We show that there exists a vertex $v$ of $F$ such that $\delta(u, v) \leq c u-\gamma$. We prove this statement by induction on the integer value $c u-\gamma \geq 0$. The statement is trivial for $c u-\gamma=0$, as we can set $v=u$. Assume $c u-\gamma \geq 1$. Since $F$ is nonempty, there exists a neighbor $u^{\prime}$ of $u$ with $c u^{\prime}<c u$ (see, e.g., [5]). The integrality of $c, u^{\prime}$ and $u$, implies $c u^{\prime} \leq c u-1$. As $c u^{\prime}-\gamma \leq c u-\gamma-1$, by the induction hypothesis there exists a vertex $v$ of $F$ such that $\delta\left(u^{\prime}, v\right) \leq c u^{\prime}-\gamma$. Therefore $\delta(u, v) \leq \delta\left(u, u^{\prime}\right)+\delta\left(u^{\prime}, v\right) \leq 1+c u^{\prime}-\gamma \leq$ $c u-\gamma$.

Given two vertices $u$ and $v$ and a face $F$ of a lattice polytope $P$, we have $\delta(u, v) \leq \delta(u, F)+\delta(v, F)+$ $\delta(F)$. By applying Lemma 2 to both $u$ and $v$, we obtain an upper bound on $\delta(u, v)$ that depends on $F$ :

Lemma 3. Let $P$ be a lattice polytope, and let $u, v$ be vertices of $P$. Let $c$ be an integral vector, $\gamma=\min \{c x: x \in P\}$, and $F=\{x \in P: c x=\gamma\}$. Then $\delta(u, v) \leq \delta(F)+c u+c v-2 \gamma$.

Let $P$ be a $(0, k)$-polytope in $\mathbb{R}^{n}$ and let $l=\min \left\{x_{i}: x \in P\right\}$ and $h=\max \left\{x_{i}: x \in P\right\}$ for some $i \in\{1, \ldots, n\}$. We can bound the distance between any two vertices $u$ and $v$ of $P$ by bounding their distances from the faces $L=\left\{x \in P: x_{i}=l\right\}$ and $H=\left\{x \in P: x_{i}=h\right\}$. If $u_{i}+v_{i} \leq l+h$, Lemma 3 applied with $F=L, c=e^{i}$ and $\gamma=l$ implies $\delta(u, v) \leq \delta(L)+(h-l)$. If $u_{i}+v_{i} \geq l+h$, Lemma 3 applied with $F=H, c=-e^{i}$ and $\gamma=-h$ implies $\delta(u, v) \leq \delta(H)+(h-l)$. Since $L$ and $H$ are $(0, k)$-polytopes of dimension at most $n-1$, we have that both $\delta(L)$ and $\delta(H)$ are at most $\delta_{k}^{n-1}$. 
Lemma 4. Let $P$ be a $(0, k)$-polytope in $\mathbb{R}^{n}$, and suppose that there exists $i \in\{1, \ldots, n\}$ such that $x_{i} \in[l, h]$ for every $x \in P$. Then $\delta(P) \leq \delta_{k}^{n-1}+(h-l)$.

Given a $d$-dimensional $(0, k)$-polytope $P$, Kleinschmidt and Onn prove the bound $\delta(P) \leq k d$ by essentially applying Lemma 1, and then Lemma 4 inductively. Therefore, their bound uses Lemma 2 only with vectors $c= \pm e^{i}$. To prove our refined bound, we will use Lemma 2 also with different vectors c. We are now ready to give the proof of Theorem 1 .

Proof of Theorem 11. Let $P$ be a $d$-dimensional $(0, k)$-polytope, with $k \geq 2$. The proof is by induction on $d$. The base cases are $d=0$ and $d=1$. The diameter of a 0 -dimensional polytope is clearly zero, and the diameter of a 1-dimensional polytope is at most one, thus also bounded by $\left.\mid k-\frac{1}{2}\right\rfloor=k-1$ since $k \geq 2$.

We now assume $d \geq 2$. Let $u, v$ be vertices of $P$. By the induction hypothesis we assume that Theorem 1 is true for $(0, k)$-polytopes of dimension at most $d-1$. In particular, $\delta_{k}^{d-1} \leq\left\lfloor\left(k-\frac{1}{2}\right)(d-1)\right\rfloor$, and $\delta_{k}^{d-2} \leq\left\lfloor\left(k-\frac{1}{2}\right)(d-2)\right\rfloor$. Thus, in order to prove the inductive step, it is sufficient to show one of the following two inequalities:

$$
\begin{aligned}
& \delta(u, v) \leq \delta_{k}^{d-1}+k-1 \\
& \delta(u, v) \leq \delta_{k}^{d-2}+2 k-1
\end{aligned}
$$

Claim 1 We can assume that $P$ is full-dimensional.

Proof of claim. By Lemma 1, there exists a full-dimensional $(0, k)$-polytope in $\mathbb{R}^{d}$ with the same 1 skeleton as $P$. $\diamond$

Claim 2 We can assume that $P$ intersects all facets of the hypercube $[0, k]^{d}$.

Proof of claim. If there exists a facet $G$ of the hypercube $[0, k]^{d}$ with $P \cap G=\emptyset$, then let $i \in\{1, \ldots, d\}$ be such that $l \leq x_{i} \leq h$, with $l \geq 1$ or $h \leq k-1$. By Lemma 4, $\delta(u, v) \leq \delta_{k}^{d-1}+k-1$, i.e. (11) is satisfied. $\diamond$

In the remainder of the paper, we will denote by $k^{d}$ the $d$-dimensional vector with all entries equal to $k$.

Claim 3 We can assume that $u+v=k^{d}$.

Proof of claim. If $u+v \neq k^{d}$, there exists an index $i \in\{1, \ldots, d\}$ such that $u_{i}+v_{i} \leq k-1$ or $u_{i}+v_{i} \geq k+1$. By Lemma 3 applied with $c=e^{i}$ or $c=-e^{i}$, respectively, we obtain $\delta(u, v) \leq \delta(F)+k-1$, where $F$ is the face of $P$ that minimizes $c x$. As $F$ is a $(0, k)$-polytope of dimension at most $d-1$, we have $\delta(F) \leq \delta_{k}^{d-1}$, therefore $\delta(u, v) \leq \delta_{k}^{d-1}+k-1$, i.e. (1) is satisfied. $\diamond$

Claim 4 We can assume that $u \in\{0, k\}^{d}$.

Proof of claim. Assume that $u$ has one component $u_{i}, i \in\{1, \ldots, d\}$, with $1 \leq u_{i} \leq k-1$. In this case we show that (2) is satisfied. Since the set $\left\{x \in P: x_{i}=0\right\}$ is nonempty, there exists a neighbor $s$ of $u$ with $s_{i}<u_{i}$ (see, e.g., [5]). By the integrality of $s$ and $u$, this implies $s_{i} \leq u_{i}-1$. Symmetrically, since the set $\left\{x \in P: x_{i}=k\right\}$ is nonempty, $u$ has a neighbor $t$ with $t_{i} \geq u_{i}+1$. If $s_{j}=t_{j}=u_{j}$ for all $j \in\{1, \ldots, d\}, j \neq i$, then by setting $\lambda=\frac{t_{i}-u_{i}}{t_{i}-s_{i}}$ we have $\lambda s+(1-\lambda) t=u$, contradicting the fact that $u$ is a vertex of $P$. Thus, there exists an index $j \in\{1, \ldots, d\}$ with $j \neq i$ such that either $s_{j} \neq u_{j}$ or $t_{j} \neq u_{j}$. Therefore there exists a neighbor $w$ of $u$ such that $w_{i} \neq u_{i}$ and $w_{j} \neq u_{j}$, for distinct indices $i, j \in\{1, \ldots, d\}$ (see Fig. 11 $(i)$ ).

We assume without loss of generality that $w_{i}<u_{i}$ (if not, we can perform the change of variable $\left.\tilde{x}_{i}=k-x_{i}\right)$. Analogously, we assume $w_{j}<u_{j}$. As $u+v=k^{d}$, we have $w_{i}+w_{j}+v_{i}+v_{j} \leq 2 k-2$. Let $\gamma=\min \left\{x_{i}+x_{j}: x \in P\right\}$ and $F=\left\{x \in P: x_{i}+x_{j}=\gamma\right\}$. By Lemma 3 (with $c=e^{i}+e^{j}$ ), $\delta(w, v) \leq \delta(F)+w_{i}+w_{j}+v_{i}+v_{j}-2 \gamma \leq \delta(F)+2 k-2-2 \gamma$ (see Fig. 11 $(i i)$ ).

We now show that $\delta(F) \leq \delta_{k}^{d-2}+\gamma$. Let $\bar{F}$ be the projection of $F$ onto the $j$-coordinate hyperplane. $\bar{F}$ is a $(0, k)$-polytope in $\mathbb{R}^{d-1}$ and, by Lemma $1, \bar{F}$ has the same 1-skeleton of $F$. Note that, for any $x \in F, x_{i}=\gamma-x_{j}$ and $x_{j} \geq 0$ imply $x_{i} \leq \gamma$. Therefore, $x_{i} \leq \gamma$ for any $x \in \bar{F}$. Then, by Lemma 4 , $\delta(\bar{F}) \leq \delta_{k}^{d-2}+\gamma$, thus $\delta(F) \leq \delta_{k}^{d-2}+\gamma$.

This implies $\delta(w, v) \leq \delta_{k}^{d-2}+2 k-2-\gamma$ and, since $\gamma \geq 0$ and $\delta(u, w)=1$, finally $\delta(u, v) \leq$ $\delta(u, w)+\delta(w, v) \leq \delta_{k}^{d-2}+2 k-1$, i.e. (2) is satisfied. $\diamond$ 
By possibly performing the change of variable $\tilde{x}_{1}=k-x_{1}$, we can further assume without loss of generality that $u_{1}=k$, and $v_{1}=0$.

Let $F$ be the face of $P$ defined by $F=\left\{x \in P: x_{1}=0\right\} . F$ is a $(0, k)$-polytope of dimension at most $d-1$, thus $\delta(F) \leq \delta_{k}^{d-1}$. By Lemma 2 (with $c=e^{1}$ ), there exists a vertex $u^{\prime}$ of $F$ such that $\delta\left(u, u^{\prime}\right) \leq k$. Observe that both $u^{\prime}$ and $v$ lie in $F$ and therefore $\delta\left(u^{\prime}, v\right) \leq \delta_{k}^{d-1}$.

If $u^{\prime}=\left(0, u_{2}, \ldots, u_{d}\right)$, then $u$ and $u^{\prime}$ are adjacent vertices of the hypercube $[0, k]^{d}$, implying that $\operatorname{conv}\left\{u, u^{\prime}\right\}$ is an edge of $[0, k]^{d}$ (see Fig. $2(i)$ ). As $P$ is convex and it is contained in $[0, k]^{d}$, it follows that $\operatorname{conv}\left\{u, u^{\prime}\right\}$ is also an edge of $P$. Therefore, $\delta\left(u, u^{\prime}\right)=1$ and consequently $\delta(u, v) \leq \delta_{k}^{d-1}+1$. As $k \geq 2$, it follows $\delta(u, v) \leq \delta_{k}^{d-1}+k-1$, i.e. (1) is satisfied.

Thus we now assume $u^{\prime} \neq\left(0, u_{2}, \ldots, u_{d}\right)$ (see Fig. $\left.2(i i)\right)$. Then, there exists an index $i \in\{2, \ldots, d\}$ such that $u_{i}^{\prime}+v_{i} \leq k-1$ or $u_{i}^{\prime}+v_{i} \geq k+1$. We assume without loss of generality that $u_{i}^{\prime}+v_{i} \leq k-1$ (if not, we can perform the change of variable $\tilde{x}_{i}=k-x_{i}$ ). Let $\gamma=\min \left\{x_{i}: x \in F\right\}, F^{\prime}=\left\{x \in F: x_{i}=\gamma\right\}$. $F^{\prime}$ is a $(0, k)$-polytope, and it has dimension at most $d-2$ because it is contained in the intersection of the two linearly independent hyperplanes $\left\{x \in \mathbb{R}^{d}: x_{1}=0\right\}$ and $\left\{x \in \mathbb{R}^{d}: x_{i}=\gamma\right\}$. It follows that $\delta\left(F^{\prime}\right) \leq \delta_{k}^{d-2}$. Then, by applying Lemma 3 to the polytope $F$ and the vertices $u^{\prime}$ and $v$, we have $\delta\left(u^{\prime}, v\right) \leq \delta\left(F^{\prime}\right)+u_{i}^{\prime}+v_{i} \leq \delta_{k}^{d-2}+k-1$. This implies $\delta(u, v) \leq \delta\left(u, u^{\prime}\right)+\delta\left(u^{\prime}, v\right) \leq \delta_{k}^{d-2}+2 k-1$, i.e. (2) is satisfied.

\section{$3 \quad$ Further directions}

Both our upper bound and the one by Kleinschmidt and Onn are not tight for $k \geq 3$. As an example, $\delta_{3}^{2}=4$, as the maximum diameter of a lattice polygon in $[0,3]^{2}$ is realized by the octagon. It seems that our approach cannot be easily refined to obtain a tight upper bound for general $k$.

An interesting direction of research is to study the asymptotic behavior of the function $\delta_{k}^{d}$. It is known that the maximum number of vertices of a 2-dimensional $(0, k)$-polytope is in $\Theta\left(k^{2 / 3}\right)$ 3, which implies the asymptotically tight bound $\delta_{k}^{2} \in \Theta\left(k^{2 / 3}\right)$. Using cartesian products of polytopes, it follows that $\delta_{k}^{d} \in \Omega\left(k^{2 / 3} d\right)$. This provides an asymptotic lower bound on $\delta_{k}^{d}$ that is a fractional power with respect to $k$ and linear in $d$. However, the best upper bound on $\delta_{k}^{d}$ is linear both in $k$ and in $d$. In other words, there is still a significant gap between the lower and the upper bound.

\section{References}

[1] H.G. Abeledo and U.G. Rothblum. Stable matchings and linear inequalities. Discrete Applied Mathematics, 54:1-27, 1994.

[2] M.L. Balinski. Integer programming: methods, uses, computation. Management Science Series A, 12:253-313, 1965.

[3] A. Balog and I. Bárány. On the convex hull of the integer points in a disc. In Proceedings of the seventh annual symposium on Computational geometry, SCG '91, pages 162-165, New York, NY, USA, 1991. ACM.

[4] N. Bonifas, M. Di Summa, F. Eisenbrand, N. Hähnle, and M. Niemeier. On sub-determinants and the diameter of polyhedra. Discrete and Computational Geometry, pages 1-14, 2014.

[5] A. Brønsted. An Introduction to Convex Polytopes. Springer-Verlag, Berlin, New York, 1983.

[6] X. Chena, G. Ding, X. Hu, and W. Zang. The maximum-weight stable matching problem: Duality and efficiency. SIAM Journal on Discrete Mathematics, 26(3):1346-1360, 2012.

[7] D. Gijswijt and G. Pap. An algorithm for weighted fractional matroid matching. Journal of Combinatorial Theory, series B, 103:509-520, 2013.

[8] P. Kleinschmidt and S. Onn. On the diameter of convex polytopes. Discrete Mathematics, 102:75-77, 1992.

[9] M. Laurent. Geometry of Cuts and Metrics. Algorithms and Combinatorics. Springer, 1997.

[10] D.J. Naddef. The Hirsch conjecture is true for (0,1)-polytopes. Mathematical Programming, 45:109$110,1989$. 
[11] D.J. Naddef and W.R. Pulleyblank. Hamiltonicity in (0,1)-polyhedra. Journal of Combinatorial Theory, Series B, 37(1):41-52, 1984.

[12] M. Padberg. The boolean quadric polytope: Some characteristics, facets and relatives. Mathematical Programming, 45:139-172, 1989.

[13] A. Schrijver. Theory of Linear and Integer Programming. Wiley, Chichester, 1986.

[14] A. Schrijver. Combinatorial Optimization. Polyhedra and Efficiency. Springer-Verlag, BerlinHeidelberg, 2003.

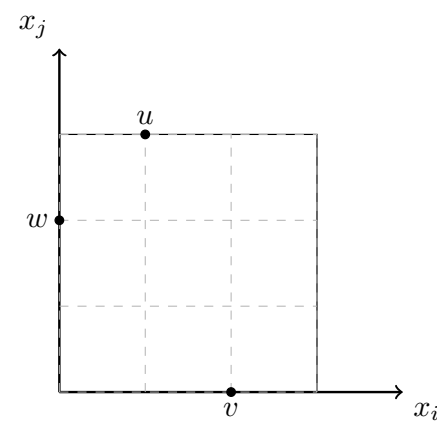

$(i)$

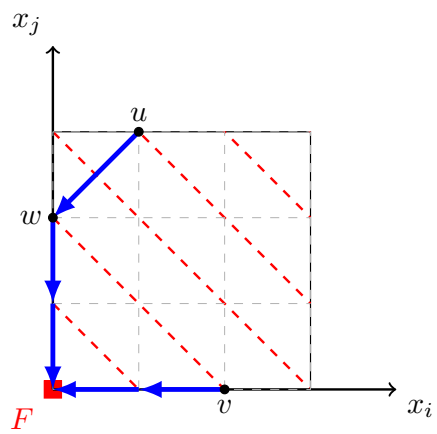

(ii)

Figure 1: In Claim 4 $(i)$ we construct a neighbor $w$ of $u$ with $w_{i}<u_{i}$, and $w_{j}<u_{j}$, (ii) we use Lemma 3 with $c=e^{i}+e^{j}$ to show that $\delta(w, v) \leq \delta_{k}^{d-2}+2 k-2$.

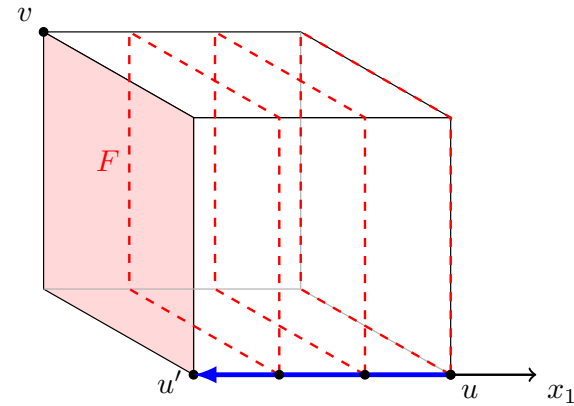

$(i)$

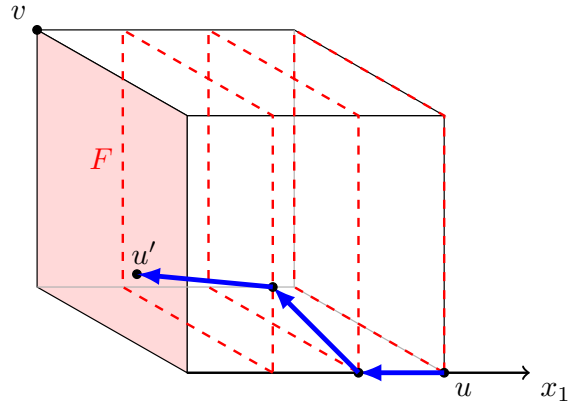

(ii)

Figure 2: To bound the distance between vertices $u \in\{0, k\}^{d}$ with $u_{1}=k$ and $v=k^{d}-u$, we construct a path from $u$ to a vertex $u^{\prime}$ with $u_{1}^{\prime}=0$. There are two cases: $(i) u^{\prime}=\left(0, u_{2}, \ldots, u_{d}\right)$, thus $\delta\left(u, u^{\prime}\right)=1$ and $\delta\left(u^{\prime}, v\right) \leq \delta_{k}^{d-1} ;(i i) u^{\prime} \neq\left(0, u_{2}, \ldots, u_{d}\right)$, thus $\delta\left(u, u^{\prime}\right) \leq k$ and $\delta\left(u^{\prime}, v\right) \leq \delta_{k}^{d-2}+k-1$. 